This document is the Accepted Manuscript version of the following article: Xianhui Che, lan Wells, Gordon Dickers, and Paul Kear, 'TDMA frame design for a prototype underwater RF communication network', Ad Hoc Networks, Vol. 10 (3): 317-327, first available online 23 July 2011.

The version of record is available online at doi:

http://dx.doi.org/10.1016/j.adhoc.2011.07.002

(C) 2011 Elsevier B. V. All rights reserved. 


\title{
TDMA Frame Design for a Prototype Underwater RF Communication Network
}

\author{
Xianhui Che, Ian Wells, Gordon Dickers, Paul Kear \\ Swansea Metropolitan University, United Kingdom
}

\begin{abstract}
Very low frequency electromagnetic communication system is used in a small scale underwater wireless sensor network for coastal monitoring purposes, as recent research has demonstrated distinct advantages of radio waves compared to acoustic and optical waves in shallow water conditions. This paper describes the detailed TDMA and packet design process for the prototype sensor system. The lightweight protocol is time division based in order to fit the unique characteristics and specifications of the network. Evaluations are based on initial beach trial as well as modeling and simulations.

Keywords: underwater electromagnetic communications, underwater wireless sensor networks, TDMA
\end{abstract}

\section{Introduction}

The recent growing interests in monitoring aqueous environments have led to extensive research on underwater wireless sensor networks (UWSN). Given modern operational requirements and digital communications technology, previous work in [1] has re-evaluated role of radio frequencies electromagnetic (RF-EM) signals in the underwater sensor networks, where a case study was also conducted on a project entitled Automated Sensing Technology for 
Coastal Monitoring (ASTEC). The project attempted to use RF-EM waves as the communication medium. This paper will demonstrate in details the protocol and packet design regime for the prototype UWSN proposed in this case.

In order to gather data and consequently quantify the effects of coastal erosion beneath the sea surface, ASTEC project aims to create the world's first automated system for monitoring and forecasting for this purpose. Engineers have classically modeled the submerged beach profile by describing two different zones: near shore zone and offshore zone. The near shore zone is active while the offshore zone is inactive with respect to the movement of sediment, silt, sand etc. In environmental science, coastal erosions can be explicitly indicated by depth of closure (DoC) which is defined as the depth seaward where neither change in bottom elevation nor net sediment exchange between nearshore and offshore can occur significantly [2]; the contour of such closure is approximately parallel to the coastline in most cases.

The UWSN built in this project aim to gather data and consequently quantifies the effects of coastal erosion beneath the sea surface. The primary role of sensors will be to either directly or indirectly measure the movement of sea bed sediments, so as to monitor the DoC. All measurement data will be sent to one destination node (i.e. the sink) on the shore or at any other designated position. The sink will be enabled with the function of Global System for Mobile communications (GSM) which allows all the collected data to be eventually transported to a central office for processing. This enables the remote collection and processing of the data. The system will also gather data regarding the health of the sensor nodes which will also be accessible 
by the user so enabling the failed nodes to be replaced as needed.

Although the usability of underwater RF technology has been quite controversial to date, it can offer great potentials in practical applications if all the system parameters are properly tuned. Unlike in acoustic systems where data rates remain relatively constant regardless of the frequency, the data rates of underwater RF communication systems are frequency-dependent and the attenuation of an EM signal increases significantly both with frequency and distance, for instance, higher frequencies although can enable higher data rates, would result in shorter transmission range [3]. Hence, when RF technology is attempted in underwater systems, the choice of operating frequency, bit rate and sensor density requires careful deliberation during the design phase. Based on respective project requirements, each design has to uniquely balance parameters such as antenna design, transmit power, duty cycle, data bandwidth, and local noise source in order to achieve an optimized solution for the specific application. This paper presents a good case of such circumstance with all the system specifications explained.

Section 2 briefly reviews the underwater RF communication characteristics. Section 3 describes the system implementation and network scenario. Section 4 discusses the protocol scheme and key issues. Section 5 demonstrates the detailed packet design process. Section 6 reveals some information of the initial beach trial and evaluates the system with modeling and simulations, and Section 7 concludes the paper. 


\section{Review of Underwater RF Communication}

Most underwater wireless networks use acoustic wave as the transmission medium nowadays, which is a proven technology for underwater sensor applications with a transmission range of up to $20 \mathrm{~km}$ [4], but the chances of getting much more out of acoustic modems are quite remote. Acoustics yields poor performance in shallow water where the transmission can be affected by turbidity, ambient noise, salinity gradients and pressure gradients; in addition, acoustic technology can place an adverse impact on marine life [5]. Another option that may be used for underwater transmission is optical wave, but this only delivers good performance in very clear water, and requires tight alignment due to the demand for the sight and the limitation of very short transmission ranges [6]. The characteristics of optical link have made itself impractical for many underwater applications. The latest research on optical UWSN is still ongoing such as [7]. Table 1 outlines the three major underwater communication technologies in terms of benefits, limitations and requirements. Compared to acoustic and optical technologies, RF-EM has some distinct advantages that make it suitable for underwater environments.

EM propagation through water is very different from propagation through air because of the high permittivity and electrical conductivity of water medium. Propagating waves continually cycle energy between the electric and magnetic fields, hence high conduction leads to strong attenuation of electromagnetic propagating waves. Plane wave attenuation also increases rapidly with frequency. However it is interesting to consider reversal of roles between acoustic and EM technologies as passing from water to air. In air, acoustic signals are highly attenuated so hundreds of people can hold separate 
Table 1: Comparison of Underwater Wireless Communication Technologies

\begin{tabular}{|c|c|c|}
\hline & Benefits & Limitations \\
\hline RF & $\begin{array}{l}\text { Prefers shallow water; Crosses } \\
\text { air/water/seabed boundaries easily; } \\
\text { Unaffected by turbidity, salinity, } \\
\text { and pressure gradients; Works in } \\
\text { non-line-of-sight; Unaffected by } \\
\text { sediments and aeration; Immune to } \\
\text { acoustic noise; High bandwidths (up } \\
\text { to } 100 \mathrm{Mbps)} \text { at very close range. }\end{array}$ & $\begin{array}{l}\text { Susceptible to EMI; Limited range } \\
\text { through water. }\end{array}$ \\
\hline Acoustic & $\begin{array}{l}\text { Proven technology; Range up to } 20 \\
\mathrm{~km} \text {. }\end{array}$ & $\begin{array}{l}\text { Strong reflections and attenuation } \\
\text { when transmitting through } \\
\text { water/air boundary; Poor } \\
\text { performance in shallow water; } \\
\text { Adversely affected by turbidity, } \\
\text { ambient noise, salinity and pressure } \\
\text { gradients; Limited bandwidth ( } \\
\text { bps to } 20 \text { Kbps); Impact on marine } \\
\text { life. }\end{array}$ \\
\hline Optical & Ultra-high bandwidth; Low cost. & $\begin{array}{l}\text { Does not cross water/air boundary } \\
\text { easily; Susceptible to turbidity, } \\
\text { particles and marine fouling; } \\
\text { Needs line-of-sight; Requires tight } \\
\text { alignment of nodes; Very short } \\
\text { range. }\end{array}$ \\
\hline
\end{tabular}


conversations in a crowded conference hall. Radio waves have low attenuation in air so communications must be separated in frequency through careful management of the spectrum. In water the two roles reverse themselves, and it is easy to see the potential benefits of high EM spatial attenuation in a multi-user environment which enables localized communications.

EM signaling, coupled with digital technology and signal compression techniques, has many advantages that make it suitable for niche underwater applications. Comparing to acoustic and optical wave technologies, radio frequency $(\mathrm{RF})$ EM technology allows flexible deployment of underwater wireless sensor network for coastal monitoring applications, where there is a high level of sediment and aeration in the water column. First, both acoustic and optical waves cannot perform smooth transition through air-and-water interface. EM waves can cross water-to-air or water-to-earth boundaries easily; its signal follows the path of least resistance, where both air and seabed paths can extend the transmission range. Second, EM transmissions are tolerant to turbulence that are caused by tidal waves or human activities, as opposed to the case of acoustic and optical waves. Third, EM waves can work in dirty water conditions, while optical waves are susceptible to particles and marine fouling [8]. Fourth, EM operation is also immune to acoustic noise, and it has no unknown effect on marine lives. Research on underwater EM communication is currently ongoing, with examples such as $[9,10]$. Table 2 summarizes the advantages of underwater RF-EM technology.

An important consideration for underwater RF-EM performance is its multi-path propagation feature caused by the effects of the water-to-air interface. Propagation loss and the refraction angle are such that an EM signal crosses 
Table 2: Summary of Advantages of Underwater RF-EM Technology

\begin{tabular}{|c|c|}
\hline & Features \\
\hline \multirow{7}{*}{ Performance } & $\begin{array}{l}\text { Crosses water to air boundary. Long range horizontal } \\
\text { communication using air path, water-to-air or land. }\end{array}$ \\
\hline & $\begin{array}{l}\text { Multi-path less of an issue especially in shallow water } \\
\text { conditions. }\end{array}$ \\
\hline & $\begin{array}{l}\text { Frequency agile capability. No mechanical tuned parts as in } \\
\text { an acoustic system. }\end{array}$ \\
\hline & $\begin{array}{l}\text { Covert, localized communications. Using high frequency } \\
\text { carrier for high attenuation. }\end{array}$ \\
\hline & $\begin{array}{l}\text { High Joules per bit efficiency, resulting efficient system in for } \\
\text { short range and high bandwidth applications. }\end{array}$ \\
\hline & Potential for high data rates. Use of $\mathrm{MHz}$ carrier. \\
\hline & $\begin{array}{l}\text { High propagation speed, especially important for networking } \\
\text { protocols requiring headers exchange. }\end{array}$ \\
\hline \multirow{4}{*}{ Resistance } & $\begin{array}{l}\text { Unaffected by pressure gradient. Allows horizontal } \\
\text { propagation. }\end{array}$ \\
\hline & $\begin{array}{l}\text { Immune to acoustic noise. Operation unaffected by engine } \\
\text { noise of heavy work. }\end{array}$ \\
\hline & $\begin{array}{l}\text { Unaffected by low visibility. Sediment disturbed at the sea bed } \\
\text { has no operational effect while laser systems fail to operate. }\end{array}$ \\
\hline & $\begin{array}{l}\text { Immune to aerated water. Operation in surf zone, } \\
\text { communication at speed through cavitating propeller wash. }\end{array}$ \\
\hline \multirow{4}{*}{ Implementation } & $\begin{array}{l}\text { No need for surface repeater. Crosses water to air boundary } \\
\text { for a long range without a surface repeater. }\end{array}$ \\
\hline & $\begin{array}{l}\text { Distributed transducers. Radiating cables can deliver unique } \\
\text { navigation and communications functions. }\end{array}$ \\
\hline & $\begin{array}{l}\text { Compact, portable units. Small-to-medium antennas deliver } \\
\text { acceptable performance. }\end{array}$ \\
\hline & $\begin{array}{l}\text { No effects on marine animals. Effect of acoustic signals on } \\
\text { marine mammals is becoming an issue. }\end{array}$ \\
\hline
\end{tabular}


the water-to-air boundary and appears to radiate from a patch of water directly above the transmitter. The large refraction angle produced by the high permittivity launches a signal almost parallel with the water surface [11]. This effect aids communication from a submerged station to the land and between shallow submerged stations without the need for surface repeater buoys. A similar effect can also be produced at the seabed. Since the conductivity of the seabed is much lower than water, it can provide an alternative low loss, low noise, covert communications path. In many deployments, the single propagation path with the least resistance will be dominant. Should the air path or the seabed path be chosen as the dominant data path, relatively longer transmission range can be achieved as compared to the water path. Hence the multi-path propagation of electromagnetic waves can be advantageous for signal transmission in shallow water conditions.

Magnetic coupled loop antennas are the most compact practical solution for duplex submerged systems. Loop antennas are directional in nature and this property can be exploited to allow selection of a single propagation path. Alternatively, omni-directional antennas can be implemented by crossing two loops so their planes intersect at right angles. Larger loop area will always provide greater antenna gain but practical systems can be designed using relatively compact loops, for instance, a small area loop antenna has been demonstrated in [12]. Another option is to use an electric dipole antenna for lateral electromagnetic waves [13]. Research in [9] has implemented a system using a crossed uninsulated terminated dipole with a diameter of $2 \mathrm{~cm}$ and $1 \mathrm{~m}$ in length, which makes careful reciprocal orientation unnecessary, thus simplifying deployment. 




Figure 1: Scenario of a small scale sensor network

\section{System and Network Design}

\subsection{Network Scenario}

A small scale wireless sensor network has been designed with a fixed topology as shown in Fig. 1. The fundamental purpose of this sensor network is to find the depth of closure and the movement of seabed sediment around this contour. It will only be necessary to monitor sea bed movement below the low tide line and the sensors would need to be positioned either side of the closure contour, as shown in Fig. 1. This will enable a temporal picture of sedimentary movement around this contour to be constructed.

As in most other wireless sensor networks, this network scenario follows multiple sources and single destination traffic patterns. The data delivery is carried out by cycles. Within each cycle, after the data-gathering period, all nodes stay in sleeping mode until a scheduled transmission is initiated, and then the nodes will wake up and be ready for communication. Once data transmission is complete, the nodes will revert back to sleeping mode. The distance between every two adjacent sensor nodes is set to the effective 
transmission range - 40m, which means any nodes beyond the next-hop radius will not receive any data from the source node transmission. All the sensor nodes are fixed in static locations by buoys and ballasts under the water surface. In order to reduce the bottleneck transmission problem as investigated in [17], the sink node is placed in the center of the network which is above the water surface and maintains $40 \mathrm{~m}$ distance to the next-hop sensor nodes (i.e. 2 and 5).

\subsection{Sensor Nodes}

The sensor nodes are in fixed locations and capable of determining the amount of sediment that is settling on top of them using a differential pressure sensor (future systems may also incorporate other measurements such as flow, turbidity etc). The system can be deployed for many months and so can build up a detailed profile of the movement of sediment at many positions on the sea bed. Sensor data are typically gathered at a certain interval and then stored locally.

Fig. 2 shows the block diagram of an end sensor node, an intermediate sensor node and the sink, as well as the multi-path propagation between the end sensor and the intermediate sensor. Each node is battery driven. Magnetic coupled loop antennas are the most compact practical solution for duplex submerged systems as previously discussed, which is built into the designated system. A radio modem called Seatext [14] produced by WFS is employed to offer high-tolerance communications through water, air and ground. As the modem is locked to a single frequency, the transceiver needs to be implemented in a half-duplex arrangement.

As for the communication systems, in order to ensure the delivery of 


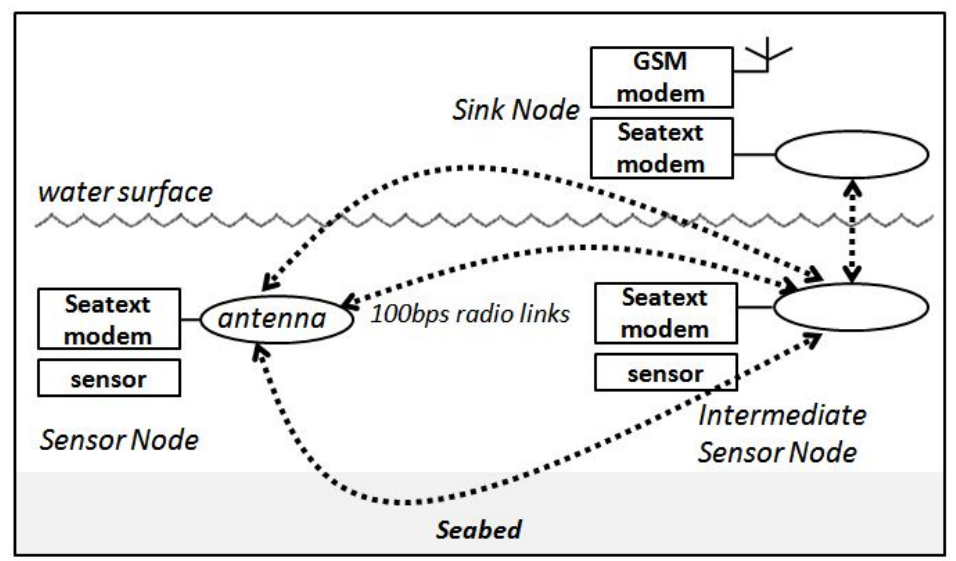

Figure 2: Schematic of the sensor node and the sink

optimized quality of signals, experiments and measurements have been carried out to determine the following settings [15]. Very low frequency (VLF) 3 $\mathrm{KHz}$ is used and the data rate is set to 100 bps. A $40 \mathrm{~m}$ radius is the achievable transmission range that can guarantee the optimized quality of signal reception, which is an improvement compared to the work in [9]. The chosen modulation technique is Binary Phase Shift Keying (BPSK). Table 3 summarizes the system specifications.

An even driven operating system called Contiki is implemented on individual nodes in the wireless sensor network [16]. The Contiki operating system has several features which are attractive to sensor network developers including a small memory footprint. It is open source based and provides both full IP networking and low-power radio communication mechanisms. For communication within a wireless sensor network, Contiki uses the lightweight Rime low-power radio networking stack [16]. The Rime stack implements sensor network protocols ranging from reliable data collection and best-effort network flooding to multi-hop bulk data transfer and data dissemination. IP packets are 
Table 3: System specifications

\begin{tabular}{ll}
\hline \hline Frequency & $3 \mathrm{KHz}$ \\
\hline Data Rate & $100 \mathrm{bps}$ \\
\hline Transmission Range & $40 \mathrm{~m}$ \\
\hline Modulation Technique & BPSK \\
\hline Coding and Error Control & RS-FEC $(255,233)$ \\
\hline Maximum Data Length & 255 bytes \\
\hline \hline
\end{tabular}

tunneled over multi-hop routing via the Rime stack. Contiki is designed for microcontrollers with small amounts of memory. A typical Contiki configuration is 2 kilobytes of RAM and 40 kilobytes of ROM. Interaction with a network of Contiki sensors can be achieved with a Web browser, a text-based shell interface, or dedicated software that stores and displays collected sensor data. The text-based shell interface is inspired by the UNIX command shell but provides special commands for sensor network interaction and sensing. Contiki was chosen for this system since it offered all the functionality required within the sensor network.

\subsection{Control Protocol Design Considerations}

Networking protocol plays a vital role in saving power and providing consistent connections in underwater wireless communications, most of which have been designed for acoustic technologies [18]. Due to the unique characteristics of the designated system, an efficient and effective control protocol needs to be carefully designed to meet the network specifications and requirements.

A few issues have to be addressed prior to the network protocol design: low data rate, half-duplex communication pattern, short transmission range, and the possible contention of resources in the burst transmission period. In this project, the short transmission range issue is not a major concern 
due to its desirable application field. $2 \times 3$ permutation with $40 \mathrm{~m}$ radius (as shown in Fig. 1) would create a plausible frontline that provides sufficient data for the coastal research [2]. The network characteristics have led to the decision of using a variation of Time Division Multiplexing Access (TDMA) as the control protocol, because it can resolve the problems addressed above. Each end sensor node will be allocated with a time slot during which it can transmit the collected data to the sink. Initially a static routing mechanism will be enabled for the data forwarding process, which is easy to implement in small scale networks. Any involved intermediate nodes also need to wake up to relay the data. Within each time slot, the source node will follow this state transition: wake up $\rightarrow$ transmitting only $\rightarrow$ sleep; while intermediate nodes will follow this transition: wake up $\rightarrow$ receiving only $\rightarrow$ transmitting only $\rightarrow$ sleep. The above is based on the assumption that no acknowledgement $(\mathrm{ACK})$ packets are facilitated in the process of communications.

There are a few advantages of using TDMA as the control protocol. First, there will be no collision between any two transmissions during the burst traffic period. The end-to-end (ETE) data delivery process for each sensor node is completed within its own time slot. Second, it fits the half-duplex transceiver mechanism. The active sensor nodes are in either transmitting-only or receiving-only mode. Third, TDMA will be used as a cross-layer control protocol which is light-weighted and efficient. Fourth, while one sensor node is transmitting data, all the uninvolved sensor nodes can stay in the sleep mode in order to conserve battery life. 


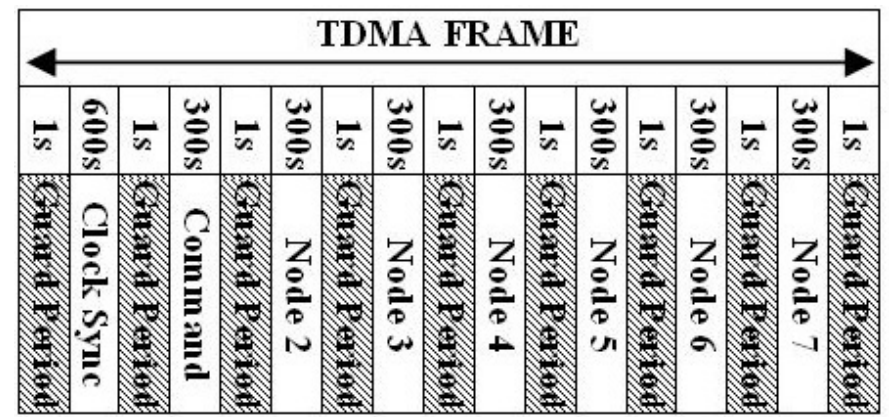

Figure 3: TDMA Frame with example slot configurations

\section{TDMA Scheme}

\subsection{TDMA Frame}

The system uses a flexible TDMA method of allocating time slots to each node in the network as a data collision avoidance mechanism. The flexibility of the system comes from the ability to define the time slot length and other parameters of the TDMA structure as desired, which enables the prototype system to experiment with different settings to obtain optimum performance. This is achieved by broadcasting a command packet, more details of which are explained in Section 5.2.

All the nodes including the sink are allocated with a time slot during which the node in question is engaged with the entire transmission process, i.e sending data, ACK and retransmission if applicable. The total time taken for all nodes to go through a transmission phase is called a TDMA frame which consists of all the time slots allocated to the nodes. Fig. 3 demonstrates an example of TDMA frame with a series of time slots that are designed for the network as shown in Fig. 1.

The data transmission is enabled with Reed Solomon (RS) $(255,233)$ 
Forward Error Correction (FEC) [19], which sets 255 bytes as the maximum size of data packets. The reason for choosing this coding technique is that it reduces hardware complexity and can still satisfy the throughput of wireless systems with restricted frequency bandwidth [20].

\subsection{System Synchronization}

In order for the system to function correctly all nodes must have a synchronized clock to make sure all slots are properly aligned. Even with a good synchronization protocol there may still be some drift of clocks between various nodes, and hence a guard band is placed between all the time slots to allow for this problem. The suggested time synchronization scheme is loosely based on the Delay Measurement Time Synchronization (DMTS) method, which has been proven to be highly energy efficient and computationally lightweight since it requires only one time broadcast to synchronize all the nodes in a network [21]. Before the data transmission commences, a one way message (i.e. synchronization packet) will be broadcasted to all sensor nodes by the sink during its time slot, which will compare the remote and the local time-stamps multiple times to obtain the line of best fit [22]. In order for the TDMA structure to accommodate transmissions of multiple time-stamps, it is important that the time synchronization packet is kept as small as possible.

Since all nodes are equipped with half-duplex communication ability, they will need to switch between receiving $(\mathrm{Rx})$ and transmitting $(\mathrm{Tx})$ mode as necessary during a time slot. While the nodes are not active, they are put into sleeping mode in order to minimize the power consumption. This is controlled by an enhanced watchdog timer on the ATmega 2560/61 micro-controller [23]. The watchdog timer gives an interrupt or a system reset when the 
counter reaches a given timeout value. This timeout period can be configured. The interrupt can be used to wake nodes from sleeping mode, and the reset mode is typically used to prevent system from hanging-up in case of runaway code.

The reset condition of watchdog timer will however cause the respective node to become unsynchronized with the other nodes so that it will loose its sense of global network time. The impact of this will amount to approximately a second. This time divergence will only affect the data time-stamps. The current data acquisition rate is in the order of hours, thus an error of 1 second in hours will not adversely affect the integrity of the data. It is improbable that a significant sediment change would occur over periods of seconds, unless a catastrophic natural disaster were to take place. The nodal time would be re-synchronized to the network just before the next data upload to the sink takes place, which is triggered by command and synchronization packets.

\subsection{Cross-Layer Addressing}

A simplified addressing scheme is used which works in a similar way as networkable protocol addressing schemes such as IP and IPX. The address is 8 bits long which identifies both logical network location and physical address, which consequently leads to a cross-layered design approach. At the

first stage of research, only static routing has been implemented to keep the system simplified. The next hop as well as the diverted next-hop information are decided in advance and embedded within each sensor's system. Within each time slot of data transmission, data packets will follow a pre-determined route to reach the sink. Dynamic routing will be worth considering if a large scale network is deployed, which is not investigated in this paper. 
Regardless of the routing technique, the end-to-end data transmission should be completed within the respective time slot of each sensor node.

The 8 bits of addressing information should be able to cover any expansion of the system that may be planned in the future. By default, the division line between network identification and node identification is exactly in the middle, i.e. 4 bits of network id and 4 bits of node id, which allows $2^{4}=$ 16 different sensor networks each supporting $2^{4}-2=14$ nodes. If future network requires different scales of networks and nodes, the division line between network id and node id can be adjusted appropriately as required.

For example, 2 bits of network id and 6 bits of node id would accommodate $2^{2}=4$ different sensor networks each with $2^{6}-2=30$ nodes. The position of the division line can be either hard-coded to the system, or dynamically determined via a network mask which is not covered in this paper.

\subsection{Data Collection and Transmission Frequency}

Depending on the data collection frequency, each time when the system is ready to transmit all the collected data through the network, there will be a certain number of readings available to be sent. Fig. 4 illustrates how this number can vary with different data gathering frequencies (e.g. every hour, every 2 hours, and so on). More frequent sensor reading exercise certainly

leads to higher accuracy for the monitoring purpose, but will burden the network with more data load.

During the data collection process, each reading will contain data from two sensor channels, which will eventually deliver the differential values required for the application. The sensors to be used in the network are estimated to produce up to 32 bits length of data. Using 16 bits to time 


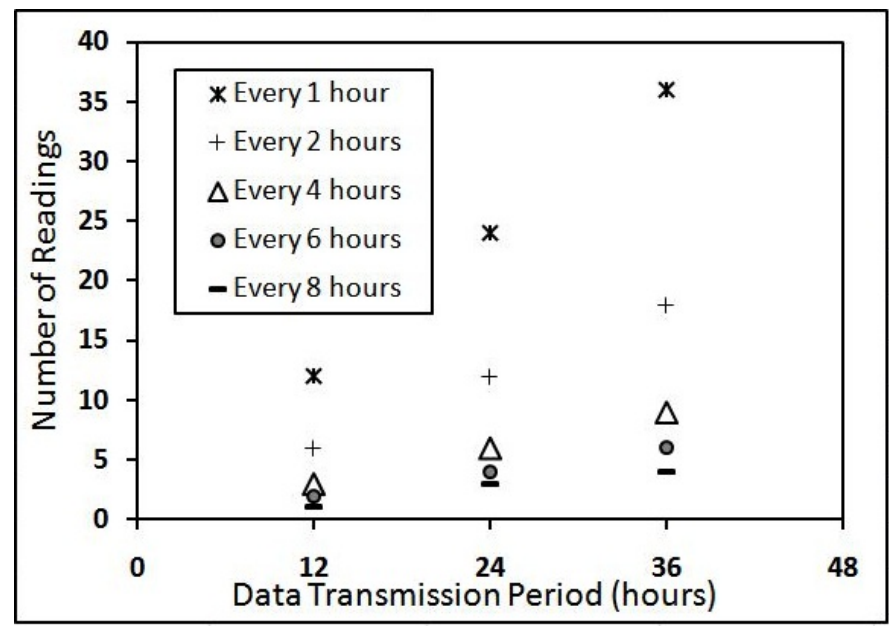

Figure 4: Number of readings with different data gathering frequencies

stamp each sensor reading, the total pressure reading data length is $32 \times 2$ $+16=80$ bits. As previously mentioned, the maximum data packet size is 255 bytes (i.e. 2040 bits). Deducting the header length 360 bits (the detailed design of header is explained later in Section 5.1), there are 1680 bits remaining for the data payload. If only one data packet per sensor is allowed for each transmission period, there can be totally 1680 /80 $=21$ readings to be accommodated within each data transmission period. Based on Fig. 4, there are some scenarios that will not be plausible to achieve, for example, if all sensors are collecting data every hour and sending the collected data to the sink every 24 hours, there will be 24 readings accumulated which outgrows the size of one data packet.

If the data packet are only partially filled, a zero data padding strategy will be used which fixes the packet to its full 255 byte size by infilling spare packet capacity with zeros. This will enable the RS(255,223) FEC algorithm to still be used for error correction. 
Table 4: Header fields of data packets

\begin{tabular}{c|lll}
\hline Types/Roles & Fields & Length & \multicolumn{1}{c}{ Details } \\
\hline \multirow{4}{*}{ Common Fields } & Addresses & 16 bits & Source and destination addresses \\
\cline { 2 - 4 } & Pkt Type & $4 \mathrm{bits}$ & Packet types: data, command, ACK, syn \\
\cline { 2 - 4 } & Reserved & $28 \mathrm{bits}$ & For future expansion \\
\cline { 2 - 4 } & CRC & $16 \mathrm{bits}$ & Cyclic redundancy check \\
\cline { 2 - 4 } & FEC & $256 \mathrm{bits}$ & Forward error control \\
\cline { 2 - 4 } & Num Read & $8 \mathrm{bits}$ & Number of sensor readings in payload \\
\hline Transmission & Num Pkts & $8 \mathrm{bits}$ & Number of packets to be transmitted \\
\cline { 2 - 4 } System Health & Pkt ID & $8 \mathrm{bits}$ & The packet number identification \\
\cline { 2 - 4 } Monitoring & RET Flag & $4 \mathrm{bits}$ & Number of retries to cross a link so far \\
\cline { 2 - 4 } & BVOLT & $8 \mathrm{bits}$ & Battery voltage of the node \\
\cline { 2 - 4 } & Wdg Flag & $4 \mathrm{bits}$ & Watchdog timer reboot flag \\
\hline
\end{tabular}

\section{Packets Design}

\subsection{Data and Acknowledgement Packets}

The sensor readings are placed in the data packet payloads to be sent to the sink. Table 4 shows the design of the data packet header fields, which forms 360 bits in length. Physical layer header is not shown here, which is 88 bits in length. The first column in Table 4 indicates the design rationale of each field.

Fields such as Addresses and Pkt Type are commonly-used fields for packet formats in network communication. The use of Num Pkts field and Pkt ID field allows multiple packets to be transported within one data transmission period, although in the beach trial and simulations later on, only one data packet is transmitted to keep the simplicity of the initial research stage. In addition, a couple of fields are designed for system health monitoring purposes, i.e. the BVOLT field logs the battery voltage of the node, and RET Flag field indicates the number of retries that a data packet has been through which indicates the health of links. Wdg Flag is a field 
to identify whether the watchdog timer has rebooted the node since the last data transmission.

The ACK packet is sent as a confirmation to the transmitting node when a data packet has been successfully received. It is 36 bits in length which contains 8 bits of destination address, 8 bits of source address, 4 bits of packet type field, and 16 bits of CRC field. Each ACK packet is only transmitted once and failure of receiving it will initiate a retransmission of the data packet.

If three failures are allowed, there can be 4 data packets and one ACK packet to be transmitted. Including the 88 bits physical layer header, the total number of bits for transmission would be $(2040+88) \times 4+(36+88)=$ 8636 bits, which would take $8636 / 100 \approx 87$ seconds. Adding one second for processing, it would be 88 seconds in total. The data timeout period is the time that a node will wait after transmitting a data packet before attempting to retransmit. This value in the worst scenario would include the time for the node to process the packet and the time for the ACK to be transmitted, i.e. $1+1.24=2.24$ which can be rounded up to $3 \mathrm{~s}$ to leave the system with reasonable margins. Therefore the maximum time for a single data packet to cross each link would be $88+9=97 \mathrm{~s}$ which can be rounded to $100 \mathrm{~s}$. The maximum number of hops is 3 in case of diverted routing, so the maximum slot size would be $3 \times 100=300 \mathrm{~s}$.

These time values have been rounded up in most cases and the actual values would vary depending on the circumstances; hence it is crucial to design a system mechanism that allows the flexibility of configuring these values as required. These long time periods are far larger than those of 
air-based communications systems, which brings unique characteristics of the designated system. Existing TDMA protocols for wireless sensor networks would not fit in this scenario as they are, which underlines why modified techniques explained in this paper are needed for this undersea network to function correctly.

\subsection{Command Packet}

The key to the flexibility of the system is the ability to define virtually every parameter of the TDMA frame structure, which is achieved by using a command packet as detailed in this section. The command packet is propagated to the network during the command time slot prior to data transmission. Table 5 shows the design of the command packet format, which forms 152 bits in length excluding the 88 bits physical layer header. The first column in Table 5 indicates the design rationale of each field.

The command packet contains some usual fields that appear in the system such as Addresses, Pkt Type. All the rest of the fields are used for network and system configurations, for example DGP and DTP define the data gathering period and data transmission period respectively. Syn Num field defines the number of synchronization phases that the DMTS synchronization protocol uses [22]. Some other notable configurations of the command packet are elaborated as follows.

- Fields Flag: It would be a waste of bandwidth if all the information were contained in all the subsequent command packets. Hence Fields Flag identifies the presence of all the configuration fields in the command packet. There are 16 bits in the Fields Flag, each of which represents 
Table 5: Fields of command packets

\begin{tabular}{|c|c|c|c|}
\hline Types/Roles & Fields & Length & Details \\
\hline \multirow{4}{*}{ Common Fields } & Addresses & 16 bits & Source and destination addresses \\
\hline & Pkt Type & 4 bits & Packet types: data, command, ACK, syn \\
\hline & Reserved & 8 bits & Reserved fields \\
\hline & $\mathrm{CRC}$ & 16 bits & Cyclic redundancy check \\
\hline \multirow{4}{*}{ System Flags } & RET Flag & 4 bits & Number of retries to cross a link so far \\
\hline & Fields Flag & 16 bits & Fields present in the command frame \\
\hline & Syn Num & 4 bits & Number of synchronization phases \\
\hline & Ver Flag & 4 bits & Network verification mode flag \\
\hline \multirow{2}{*}{ Define Retries } & RetD & 4 bits & Number of retries a data packet is allowed \\
\hline & RetT & 4 bits & Number of retries a syn packet is allowed \\
\hline \multirow{2}{*}{$\begin{array}{c}\text { Define Time } \\
\text { Slots }\end{array}$} & BBS & 8 bits & Building block size \\
\hline & Time Slots & 20 bits & Length of time slots \\
\hline \multirow{5}{*}{ Define Periods } & DGP & 4 bits & Data gathering period in hours \\
\hline & DTP & 8 bits & Data transmission period in hours \\
\hline & Data Tout & 8 bits & Timeout period after a data pkt is sent \\
\hline & Com Tout & 8 bits & Timeout period after a command is sent \\
\hline & Wdg Tout & 4 bits & Timeout period of watchdog timer \\
\hline \multirow{2}{*}{ Fault Mode } & Fault Flag & 8 bits & Defines if a node is to enter Fault Mode \\
\hline & Flt Per & 4 bits & Time period in the Fault Mode \\
\hline
\end{tabular}

a field in the frame. The Addresses, Pkt Type and CRC fields are not taken into account as they are mandatory for all command packets.

- Network Verification Mode: This mode is designed to verify network status upon system deployment. When the Ver Flag is set to 1 in the command packet, the network will enter into the verification mode. Dummy data packets carrying no payload are sent to the sink to test the network functionality. The verification mode will be exited by sending out a normal command packet during the next available command slot in the TDMA Frame.

- Define Retries: The RetD and RetT fields define respectively the number of retries that data packets and synchronization packets are allowed to 
attempt when they fails to reach the destination. As for the command packet, there is no FEC facility, but due to its high priority the command packet will be retransmitted indefinitely by the application software until an ACK is received from the sink node.

- Define Time Slots: This facility allows experimentation with different slot sizes for the TDMA frame when the system is operational. The start of each time slot will be a relative time value that is used by the nodes. The time slot sizes are determined by defining a smaller unit called Building Block Size (BBS) which is 8 bits in size, and each time slot will begin after a multiple times of the BBS value. The Time Slots field consists of four subfields which are 4 bits for the clock synchronization, 4 bits for the command packet, 8 bits for the data slots, and 4 bits for the guard band. All subfields define the multipliers used for the building block time found in the BBS field, except that the guard subfield uses a default block size of 0.5 second. For example, if the Time Slots field had the entries of $6,3,3,2$ with a value of 100 seconds in the BBS field, then this would produce a TDMA frame with the slot sizes as shown in Fig. 3 .

- Fault Mode: This mode is only used for node testing purpose. The Fault Flag field contains 8 bits which identifies fault mode disabled mode, fault mode not used mode, and flags for the six nodes. Should more sensor nodes are deployed in the future, this field can be expanded by using the Reserved field. The Flt Per indicates the time period that the node in question is to be switched off for in the fault mode. 


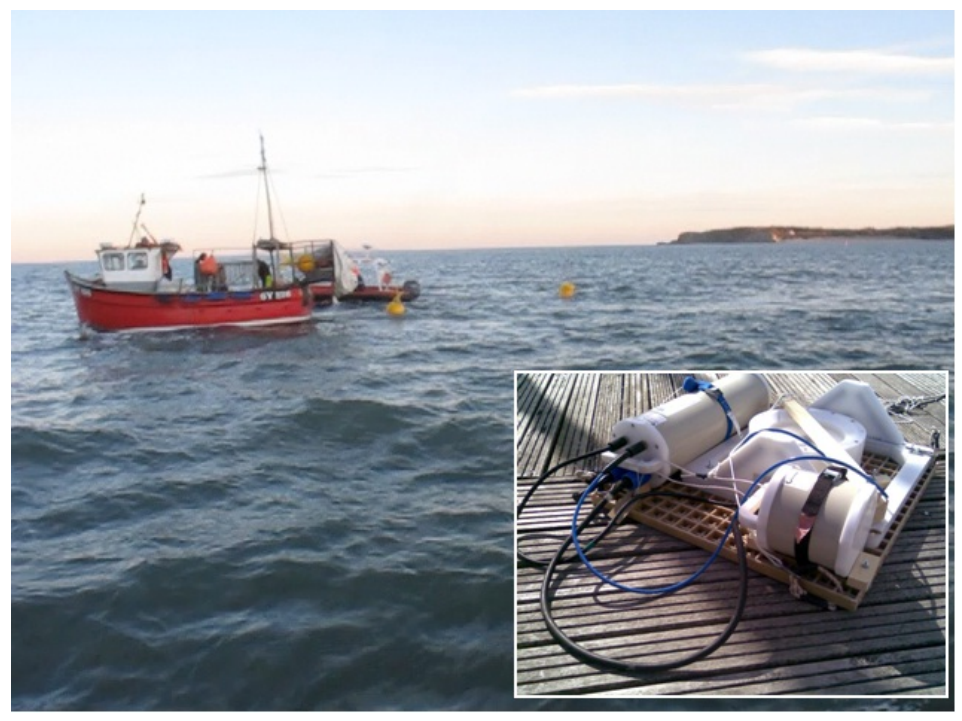

Figure 5: Sensor node part for the beach trial

\section{Evaluation and Analysis}

\subsection{Initial Beach Trial}

Figure 5 shows the sensor node part to be deployed on the seabed. Each node logged 2-channel pressure sensor data along with the logging time stamp. These pressure sensor readings and time stamps were subsequently transmitted to the sink or Master node. Data from all sensors and all nodes were relayed to the remote computer. The data clearly shows two tidal cycles during the 24 hours period. Data was not received by the cental office computer between hours 18 and 24 due to it conducting a routine system backup, although it is believed that the network was still operational during this time.

Pressure measurements from each pressure sensor on each node are collected in the unit of Pascals $(\mathrm{Pa})$ which can be converted to Bars by dividing by $1 \times 105$ later on. It is worth noting that the pressure sensors used in this trial 


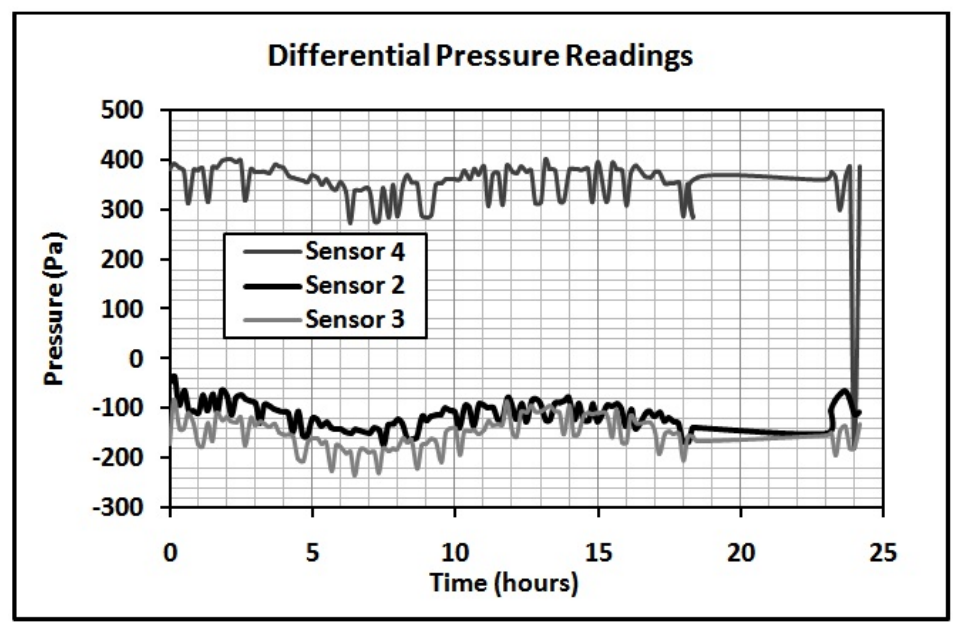

Figure 6: Differential pressure readings from the trial

were gauge rather than absolute pressure sensors. Gauge pressure sensors takes atmospheric pressure into account before any reading is taken and therefore to convert these readings to absolute pressure values requires adding 1 Bar or 1 x105 Pa to the collected plots. With this in mind the variation in pressure between approximately 0.2 and 0.55 bar would equate to a sea depth of around $2-5.5 \mathrm{~m}$. The actual reading that allows calculation of sediment depth is the difference between the two pressure sensor channels, one channel measuring hydrostatic pressure and the other measuring the weight of sediment and water. It is clear that the same differential pressure will be calculated from either a pair of gauge pressure sensors or a pair of absolute pressure sensors.

The difference between pressure sensor readings at each node has been calculated and is shown in Figure 6. The pressure difference is used as a measure of sediment transport. In this figure the pressure differences exhibit noise fluctuations. It is not expected that significant sediment transport 
occurred during the 24 hours trial and thus the above represents the sensitivity level of the system. The initial trial has demonstrated the target project deliverable of a wireless network of real-time subsea erosion sensors. Further, longer term trials are envisaged now that the network has been proven.

\subsection{Simulation and Discussions}

The network structure as shown in Fig. 1 has been modeled in the virtual environment to evaluate the network performance of the designated protocol. In order to deliver efficient and effective service, the network protocol should account for the following parameters: delay, battery consumption, throughput, and so on. OPNET Modeler is used as the simulation platform [24], within which the original source codes for propagation delay model (dra_propdel.ps), transmission delay model (dra_txdel.ps) and power attenuation model (dra_power.ps) have been altered to simulate underwater EM communication environment. These parameters are customized based on the following equations [1], where $\lambda$ is the wavelength; $\mathrm{f}$ is the frequency; $\sigma$ is the conductivity; $\mathrm{v}$ is the propagation velocity; $\delta_{\text {skin }}$ is the skin depth.

$$
\begin{gathered}
\lambda=1 / \sqrt{f \sigma \times 10^{-7}} \\
v=\sqrt{f \times 10^{7} / \sigma} \\
\delta_{\text {skin }}=1 /\left(2 \pi \sqrt{f \sigma \times 10^{-7}}\right)
\end{gathered}
$$

The system is set up according to the network specifications, as shown in Table 6. Each sensor node should have four states: data-gathering, transmitting, receiving, and idle (i.e. sleeping). The power consumption 
Table 6: SIMULATION SETUP PARAMETERS

\begin{tabular}{lll}
\hline \hline Data Tx/Rx Rate & 100 bits/second & \\
\hline Time Slot & 300 seconds & \\
\hline Burst Inter-arrival Time & 2000 seconds & \\
\hline Power Consumption Rate & Tx State: & $520 \mathrm{~mA}$ (measured) \\
& Rx State: & $210 \mathrm{~mA}$ (measured) \\
& Idle State: & $0.2 \mu \mathrm{A}$ (estimated) \\
\hline \hline
\end{tabular}

Table 7: Delay and Goodput Measurements (seconds)

\begin{tabular}{l|llllll|l}
\hline \hline Packet & \multicolumn{2}{|l}{ Node } & Node & Node & Node & Node Node & Goodput \\
Size & $\mathbf{4}$ & $\mathbf{2}$ & $\mathbf{6}$ & $\mathbf{5}$ & $\mathbf{3}$ & $\mathbf{7}$ & \\
\hline 360 & 307.2 & 603.6 & 907.2 & 1207.2 & 1503.6 & 1807.2 & $0 \%$ \\
\hline 1100 & 322.0 & 611.0 & 922.0 & 1222.0 & 1511.0 & 1822.0 & $76 \%$ \\
\hline 2040 & 340.8 & 620.4 & 940.8 & 1240.8 & 1520.4 & 1840.8 & $82 \%$ \\
\hline \hline
\end{tabular}

for each state is either measured or estimated on the actual node system, except for the data gathering state which is estimated to be negligible hence is excluded in the simulations.

As for the packet size, three configurations are chosen: 2040 bits (maximum data payload), 1100 bits (half size data payload), and 360 bits (no data payload, header only). The hypothesis was that the bandwidth cost for the overheads will lower down the network efficiency, hence the data payload size should be maximized in order to achieve high network goodput. The goodput measurements shown in Table 7 imply that as long as data payload occupancy is over $50 \%$ of the field, the network will deliver acceptable efficiency (i.e. more than $76 \%$ goodput).

All the data can be effectively delivered to the sink since no node failure is simulated. Table 7 shows various total delays for different data packet size. Total delay includes end-to-end transmission delay and the waiting time prior to transmission. The waiting time consists of the duration for 


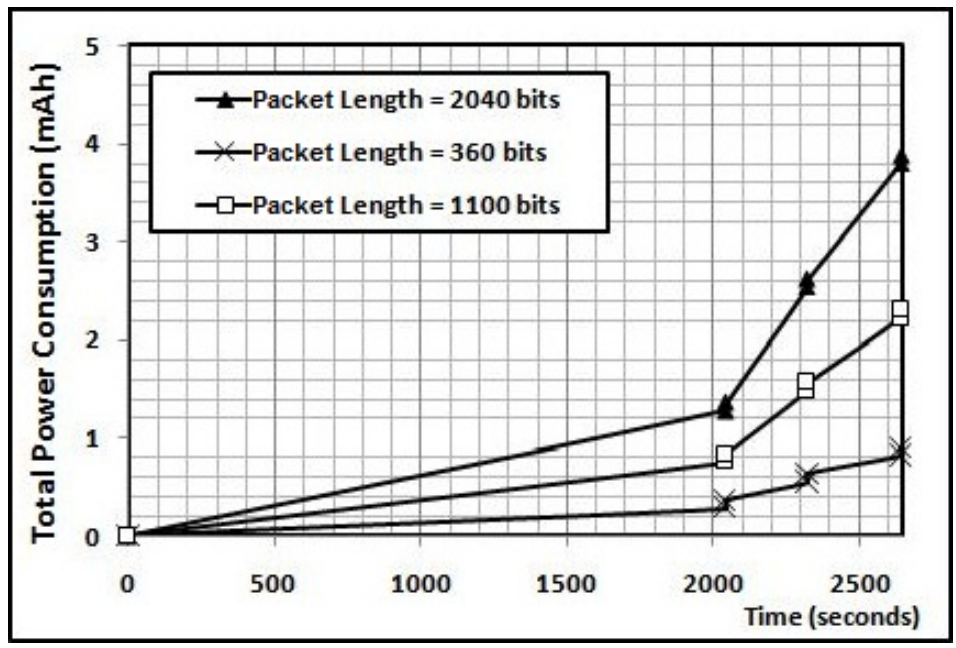

(a) Sink node

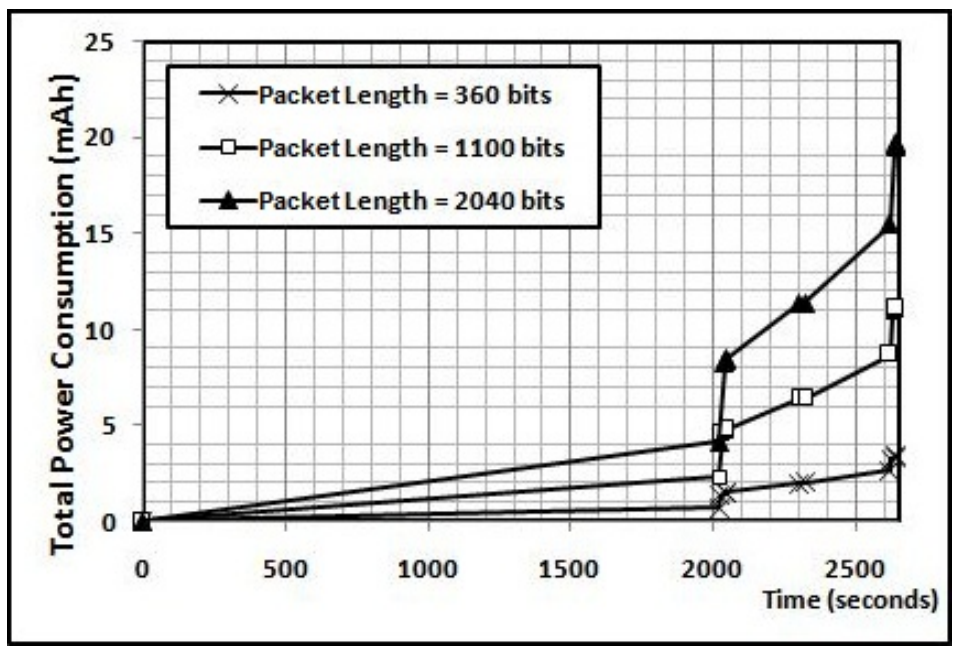

(b) Intermediate Node

Figure 7: Total power consumption for one cycle 
synchronization and command packets to cross the network, as well as the pending time for the allocated slot. The results indicate that the total networking time for one cycle transmission lasts roughly half an hour. The variation of packet size generates some fluctuation of network delay in the order of seconds; however this jittering is not significant to impair the network performance, since the delay is measured in minutes in the context of this system.

Battery usage is an important performance parameter in the UWSN, which indicates the energy efficiency of the network protocol. Fig. 7 shows the total power consumption of the sink and an intermediate node (node 2 or 3 in Fig. 1) for one TDMA cycle, which compares the power usage for different scenarios - long, medium, and short data packet size. The statistics indicate that longer data payload results in more battery usage; however the increase of data payload size does not remarkably affect the power consumption of the sink node, in other words, the difference between minimum and maximum scenarios of data payload size is minimal. The excessive increase of power consumption of intermediate nodes is substantial, which will impose limitations on the battery deployment.

Fig. 8 compares the total power consumption of the sink, an end sensor node, and an intermediate sensor node for two TDMA transmission cycles where the maximum packet size is used. The intermediate node consumes more power than the sink node which was not expected. The explanation for this occurrence follows. Even though the sink node needs to stay awake for each time slot, the power for receiving data is much lower than the power needed for sending data. On the other hands, the intermediate nodes need 


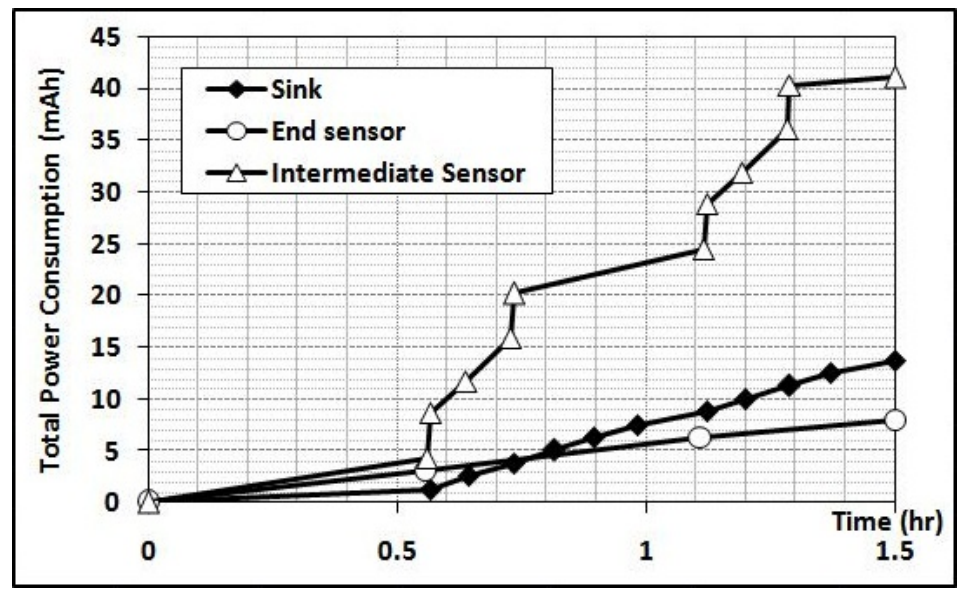

Figure 8: Total power consumption for two cycles

to be engaged with the transmission states three times in a TDMA cycle: one time to transmit its own data, and two times to relay its neighbor's data. Hence the overall power usage for the intermediate nodes will exceed the one for the sink. Assuming that a compact and low-cost 18Ah battery is employed for each node, and that two cycles of readings will be sent daily, the battery capacity will be able to maintain the network for approximately one year. This demonstrates a plausible deployment of the designated system.

When using RF technology in underwater scenarios, each solution has to uniquely balance parameters such as antenna design, transmit power, duty cycle, data bandwidth, antenna size, and local noise sources in order to achieve an optimized solution for the specific application. Given that the attenuation of an EM signal increases significantly with both frequency and distance, the choice of operating frequency, bit rate, and sensor density requires careful deliberation during the design phase. 


\section{Conclusion}

A small scale underwater wireless sensor network is designed to employ RF electromagnetic communication of which the capabilities have been re-evaluated in previous research. The network has a multi-hop static topology for coastal monitoring purposes which will work under shallow water conditions. TDMA is chosen as the control protocol for the half-duplex pattern communication network. This paper described the details of the MAC layer protocol and the use of a flexible TDMA framing technique that was used to gather the data from the prototype sensor network. The basic aim of designing the prototype was to maintain as much flexibility as possible because of the many unknowns in the hostile deployment environment. The initial beach trial demonstration and the analysis of the simulation results have led to the conclusion of feasibility and effectiveness of the designated system and network architecture. Future research should further explore the different aspects of network performance, such as scalability, necessity for reliable transmission, and comparing the chosen protocol with other potential options.

\section{References}

[1] X. Che, I. Wells, G. Dickers, P. Kear, X. Gong, (2010). Re-Evaluation of RF Electromagnetic Communication in Underwater Sensor Networks, IEEE Communications Magazine, 48(12), 143-151.

[2] M. R. Phillips, and A. T. Williams, (2007). Depth of Closure and Shoreline Indicators - Empirical Formulae for Beach Management, J. Coastal Research, 23, 487-500. 
[3] R. Gabillard, P. Degauque, and J. R. Wait, (1971). Subsurface Electromagnetic Telecommunication - a Review, IEEE Trans. Communications, COM-19(6), 1217-1228.

[4] J. Heidemann, W. Ye, J. Wills, and A. Syed, (2006). Research Challenges and Applications for Underwater Sensor Networking, Proc. IEEE Wireless Communication and Networking Conf.

[5] W. Au, P. Nachtigall and J. Pawloski, (1977). Acoustic Effects of the ATOC signal $(75 \mathrm{~Hz}, 195 \mathrm{~dB})$ on Dolphines and Whales, J. Acoustical Society of America, 101, 2973-2977.

[6] F. Schill, U. Zimmer, and J. Trumpf, (2004). Visible Spectrum Optical Communication and Distance Sensing for Underwater Applications, Proc. Australasian Conf. Robotics and Automation.

[7] D. Anguita, D. Brizzolara, and G. Parodi, (2009). Building an Underwater Wireless Sensor Network based on Optical Communication: Research Challenges and Current Results, Proc. 3rd IEEE Int. Conf. Sensor Technologies and Applications, 476-479.

[8] A. Ken, M. J. Smith, and M. J. Cowling, (2003). Optimizing Optical Port Size on Underwater Marine Instruments to Maximize Biofouling Resistance, Materials an Design, 24(4), 247-253.

[9] U. M. Cella, R. Johnstone, and N. Shuley, (2009). Electromagnetic Wave Wireless Communication in Shallow Water Coastal Environment: Theoretical Analysis and Experimental Results, Proc. 4th ACM Int. Workshop on UnderWater Networks. 
[10] X. Che, I. Wells, P. Kear, G. Dickers, X. Gong, and M. Rhodes, (2009). A Static Multi-Hop Underwater Wireless Sensor Network Using RF Electromagnetic Communications, Proc. 2nd IEEE Int. Workshop on Specialized Ad Hoc Networks and Systems, 460-463.

[11] D. W. White, (1975). Investigation of an Underwater Electromagnetic Communications Channel, Masters Dissertation, Dept. Elect. and Comp. Sci., Cambridge, 2-35.

[12] P. Smith, (2008). Measurement and Analysis of Transmit Antenna Configurations for Underwater RF Communications, Proc. 3rd SEAS DTC Technical Conf.

[13] R. King, (1989). Lateral Electromagnetic Waves from a Horizontal Antenna for Remote Sensing in the Ocean, IEEE Transactions on Antennas and Propagation, 37, 1250-1255.

[14] I. Crowther, (2009). Through-Water and Through-Ground Radio Modem: Seatext, KM Seatext E\&I, Wireless Fibre Systems.

[15] M. Rhodes, B. Hyland and D. Wolfe, (2006). Underwater Communications System, U.S. Patent 134331.

[16] A. Dunkels, B. Gronvall, and T. Voigt, (2004). Contiki - A lightweight and flexible operating system for tiny networked sensors, Proceedings of the First IEEE Workshop on Embedded Networked Sensors, Tampa, Florida, USA.

[17] X. Che, I. Wells, P. Kear, G. Dickers, X. Gong, and M. Rhodes, (2009). A Static Multi-Hop Underwater Wireless Sensor Network Using RF 
Electromagnetic Communications, Proc. 2nd IEEE Int. Workshop on Specialized Ad Hoc Networks and Systems, 460-463.

[18] D. Pompili, and I. F. Akyildiz, (2009). Overview of Networking Protocols for Underwater Wireless Communications, IEEE Communications Magazine, 97-102.

[19] A. Rashid, F. Fitzek, O. Olsen, Y. Moullec, and M. Gade, (2006). A Low Complexity, High Speed, Regular and Flexible Reed Solomon Decoder for Wireless Communication, IEEE Design and Diagnostics of Electronic Circuits and Systems, 31-36.

[20] N. Jiang, K. Peng, and Z. Yang, (2008). A Low Complexity Reed-Solomon Decoder, Proc. 4t IEEE Int. Conf. on Circuits and Systems for Communications, 288-292.

[21] I. Rhee, J. Lee, J. Kim, E. Serpedin, W. Erchin, and Y. Wu, (2009). Clock Synchronization in Wireless Sensor Networks: An Overview. Sensors, 9, 56-85.

[22] S. Ping, (2003). Delay Measurement Time Synchronization for Wireless Sensor Networks, Intel Research, IRB-TR-03-013.

[23] 8-bit Atmel Microcontroller with 64k/128k/256k Bytes In-System Programmable Flash, available at www.atmel.com.

[24] OPNET Modeler, http://www.opnet.com. 